\title{
Correlation of contrast sensitivity with the severity of primary open angle glaucoma
}

\author{
Satyam Kumar Jha', Ashwini K. V',* \\ ${ }^{1}$ Optometrist, ${ }^{2}$ Associate Professor, Dept. of Ophthalmology, Sapthagiri Institute of Medical Sciences and Research Centre, \\ Bangalore, Karnataka, India \\ *Corresponding Author: Ashwini K. V \\ Email: ashwini_kulenur@yahoo.com
}

\begin{abstract}
Aims: To assess the contrast sensitivity (CS) in different stages of primary open angle glaucoma and to correlate it with the severity (mild, moderate \& severe) of glaucoma.

Materials and Methods: This prospective randomized study was conducted at Sapthagiri institute of medical sciences and research centre, Bangalore between June 2017 \& December 2017. A total of 100 eyes of 55 individuals aged 25-80 years diagnosed with POAG were enrolled in this prospective study. Selected subjects underwent detailed ophthalmic examination including ocular history, slit lamp examination, objective \& subjective refraction (if required) to obtain best corrected visual acuity (BCVA) on Snellen visual acuity chart and dilated fundus evaluation using indirect ophthalmoscopy. Eyes with clear media and $\geq$ BCVA $6 / 60$ were included and contrast sensitivity was measured using Pelli Robson chart at 1 meter distance. HFA report of included subjects were collected from Glaucoma department to classify them in to mild, moderate and severe groups according to their visual field findings (pattern deviation of HFA report). Classification was done according to International statistical classification of disease and related health problem (ICD 10). One way ANOVA and t-test was used to analyze the data.

Results: There was 36\% mild, $26 \%$ moderate and 38\% severe cases of POAG classified according to ICD 10. The mean value of contrast in mild, moderate and severe POAG was $1.4341,1.3267$ and 1.1326 respectively. An analysis of variance tested whether there were significant difference in contrast sensitivity among mild, moderate and severe POAG. Results indicated that these means differed significantly $\mathrm{p}<0.01$. Hence there is gradual decrease in CS with the severity of glaucoma.

Conclusion: There was decrease in contrast sensitivity with the severity of POAG. Thus the contrast sensitivity testing should be made mandatory in evaluating POAG patients which could help in screening/diagnosis (early detection) and to assess the progression of the disease as well as response to the treatment.
\end{abstract}

Keywords: Contrast Sensitivity, Primary open angle glaucoma, Visual fields.

\section{Introduction}

Contrast sensitivity is gaining popularity as an adjunct to perimetry for management of glaucoma patients. Contrast sensitivity (CS) is very useful in two areas of glaucoma evaluation. Data now shows glaucoma patients exhibit abnormal contrast sensitivity and this abnormality can be used to assess the presence or progression of the disease. Following treatment, 60 to $70 \%$ of the patients show clinically significant improvements in CS. Contrast sensitivity can be used to evaluate the patient response to the treatment or to a change in therapy.

Contrast sensitivity test is quick and simple procedure which provides more sensitive measurements of subtle vision loss than visual acuity in glaucoma patients. Numerous studies have shown that contrast sensitivity provides useful information about functional vision that is not provided by visual acuity \& visual field. ${ }^{1-2}$ Thus using contrast sensitivity in combination with visual acuity and visual field gives a better idea about functional vision ${ }^{1}$ and may help in early detection of POAG.

It has been well demonstrated that contrast sensitivity in visual function is affected in glaucoma. ${ }^{3-5}$ Numerous reports have indicated that contrast sensitivity does seem to be selectively affected by the glaucoma process to a greater extent than is Snellen (high contrast) visual acuity. However, most of these studies have concentrated only on investigations as to whether it would be possible to detect glaucoma in patients with various contrast sensitivity tests prior to visual field damage. ${ }^{6}$ several studies have shown that CS measured were depressed in POAG group as compared to age matched normal group ${ }^{7,8}$ but the population investigated was in the early stage of disorder. Several studies have evaluated the difficulty experienced by glaucoma patients in performing visually dependent daily activities and thus compromising the quality of life. It was found that near visual acuity, visual field, and contrast sensitivity measures, are the best predictors for assessing the quality of life in glaucoma patients. ${ }^{9}$

In this study we have tried to quantitate this loss in CS related to their glaucoma damage according to their severity by use of the Pelli Robson chart and related visual field performance on the Humphrey visual field analyzer so that it may help in the early detection of POAG in new cases and to assess the progression of the disease in known cases of POAG.

\section{Materials and Methods}

This prospective cross sectional non interventional study was conducted at Sapthagiri institute of medical sciences and research centre, Bangalore between June 
2017 to December 2017. Institutional ethical clearance was obtained from institutional ethical committee. The study population were the diagnosed cases of POAG visiting glaucoma department. A total of 100 eyes of 55 individuals aged 25-80 years diagnosed with POAG were enrolled in this prospective study. Clinical condition in which CS can be reduced while VA remains normal such as refractive surgery, minimal capsular opacification, heavy smoking, multiple sclerosis, diabetics with no background retinopathy, optic neuritis, papilledema, visual pathway lesions, Parkinson's disease, Alzheimer's disease were excluded in this study and clinical condition in which contrast sensitivity \& visual acuity both are reduced such as cataract and age related maculopathy were also excluded in this study.

Informed consent was taken from the selected subjects. Selected subjects underwent detailed ophthalmic examination including ocular history, slit lamp examination, objective \& subjective refraction (if required) to obtain best corrected visual acuity (BCVA) on Snellen visual acuity chart and dilated fundus evaluation using indirect ophthalmoscopy. Eyes with clear media and $\geq$ BCVA 6/60 were included and contrast sensitivity was measured using Pelli Robson chart at 1 meter distance. All patients underwent visual field charting using Humphrey field analyzer. HFA report was used to classify them into mild, moderate and severe groups according to their visual field findings (pattern deviation of HFA report). Classification was done according to International statistical classification of disease and related health problem (ICD 10). ${ }^{12}$ One way ANOVA and t-test was used to analyze the data.

\section{Statistical Analysis}

Data entry and analyzation were done using IBM SPSS $23^{\text {rd }}$ version one way ANOVA testing and t-test was used to analze the data.

\section{Results}

In this study 100 eyes of 55 subjects were evaluated. Among them 19 were Female and 36 were Male (Graph 1). The mean age of the subjects was $61.63 \pm 10.80$ years.

\section{Graph 1}

Gender Distribution Chart

$$
34.38 \%
$$

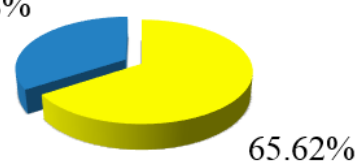

Male Female
Graph 2: Number of newly diagnosed and under treatment population

Old Vs newly Diagnosed Distribution

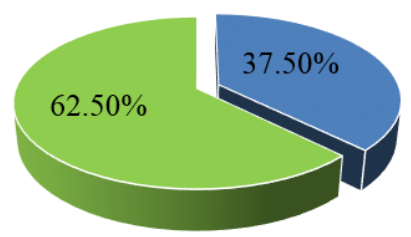

- Newly Diagnosed $\quad$ - Under Rx

Graph 2 shows among 55 subjects, 34 were old case of POAG and were taking Anti Glaucoma Medication and 21 were newly diagnosed on the day of study.

Graph 3: Distribution chart of mild, moderate \& severe POAG

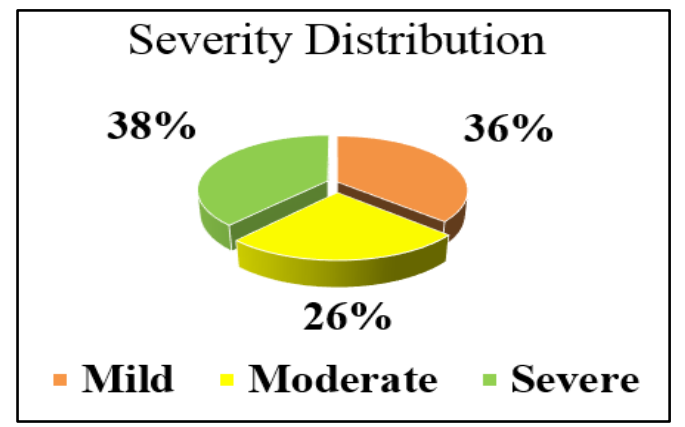

Graph 3 shows out of 100 eyes 36(38\%) eyes were mild, $26(26 \%)$ were moderate and $38(38 \%)$ were severe case of POAG.

\section{Graph 4: Comparison of mean CS in mild, moderate} \& severe POAG

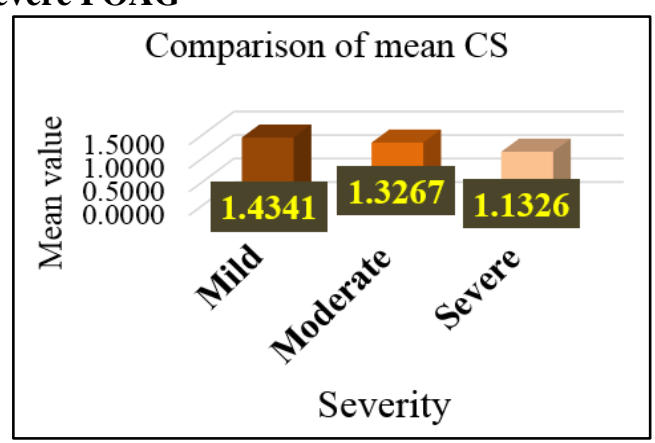

Graph 4 shows, there is gradual decrease in CS according to severity with the mean value of 1.4341 , 1.3267 and 1.1326 in mild, moderate and severe cases respectively.

An analysis of variance tested whether there were differences in significance among mild, moderate and severe POAG. Results indicated that these means differed significantly $(\mathrm{p}<0.01)$. 
The mean value of CS measured in mild POAG is 1.4341 with SD of \pm 0.1960 \& the mean value of CS measured in moderate POAG is 1.3267 with SD of \pm 0.2499 . The result shows that there is no significant difference in CS between mild \& moderate POAG. (Unpaired t test, $\mathrm{P}=0.151$ ).

The mean value of CS measured in mild POAG is 1.4341 with SD of \pm 0.1960 \& the mean value of CS measured in severe POAG is 1.1326 with SD of \pm 0.2304 . The result shows that there is significant difference in CS between mild \& severe POAG (p value $<0.05)$.

The mean value of CS measured in moderate POAG is 1.3267 with SD of \pm 0.2499 and the mean value of CS measured in severe POAG is 1.1326 with $\mathrm{SD}$ of \pm 0.2304 . The result shows that there is significant difference in CS between moderate and severe POAG. $(\mathrm{P}=0.019)$.

\section{Discussion}

In a study done by Wood JM, Lovie-Kitchin et al Contrast sensitivity measurement used in the detection of primary open angle glaucoma found CS measured were depressed in POAG group as compared to age matched normal group. The limitation of the study was that the population investigated was in the early stage of disorder. ${ }^{7}$ In an another study done by Malathi et $\mathrm{al}^{8}$ compared the CS measurements in established primary open angle glaucoma, glaucoma suspects and age matched controls. In these studies the younger subjects scored better than the older, indicating a depression of contrast sensitivity with increasing age. However, most of these studies have concentrated only on investigations as to whether it would be possible to detect glaucoma with various contrast sensitivity tests prior to visual field damage.

Another study done in India, Comparison of contrast sensitivity, visual acuity, and Humphrey visual field testing in patients with glaucoma, done by $\mathrm{W} T$ Jacob \& H Anjali found significant correlation between the mean deviation on the Humphrey perimeter and the contrast sensitivity score on the Pelli-Robson charts. The limitation of the study was that result is probably affected by age factor since they have taken older age for their study. And also they have not classified glaucoma in different stage according to their severity. ${ }^{10}$ In an another study by Hawkins A, Szlyk J, et al found that reduced contrast sensitivity is significantly correlated with visual field losses in patients with glaucoma and a visual acuity of 20/40 or better. The study data support that compared with visual acuity, the disease process preferentially affects contrast sensitivity. ${ }^{11}$

In a study by McKendrick $A$ et al explored the effects of glaucoma and aging on Low-spatialfrequency contrast sensitivity. They found older control subjects demonstrated reduced sensitivity relative to the younger group. Glaucoma group demonstrated further sensitivity reduction relative to older control subjects across the spatial frequency range for both the steadyand pulsed-pedestal tasks. Limitation is that they are unable to quantify the decreased CS according to severity of glaucoma. ${ }^{12}$

It has been found that $\mathrm{M}$ (meganocellular) cells and $\mathrm{K}$ (koniocellular) cells, types of retinal ganglion cells are responsible for contrast sensitivity. ${ }^{13} \mathrm{M} \& \mathrm{~K}$ cells are morphologically larger. There is histologic evidence for a reduction in the proportion of larger retinal ganglion cells (RGCs) in glaucomatous eyes i.e. M \& K RGCs. Several laboratories provide evidence for cell shrinkage before cell death in experimental glaucoma. ${ }^{13-14}$ hence, people with glaucoma should show dysfunction on tasks mediated by $\mathrm{M}$ or $\mathrm{K}$ neurons i.e. decreased CS. ${ }^{15}$

Several studies done on Contrast sensitivity measurement in the detection of primary open angle glaucoma found decrease in CS in POAG patients. In this study we have tried to correlate whether the increase in severity of POAG has any effect on CS. In this study, there was gradual decrease in CS with severity. There was statistical difference between different stages. Our study reflects a significant difference $(<0.01)$ in overall comparison and mild to severe POAG groups $(\mathrm{p}=<0.05)$ and moderate to severe $(\mathrm{p}=0.019)$ shows significant loss. There was no statistical difference between mild to moderate POAG. This may be because the damage in RGC in both mild and moderate POAG are very minimal as compared with severe POAG cases.

Aging has been shown to decrease CS. ${ }^{11}$ As most of the subjects in this study were above the age of 50 years $(84.38 \%)$ this may have affected the results of this study. Hence, study can be done in age matched groups to overcome the age related decrease in CS. Only POAG subjects were enrolled in this study. Another study can be done enrolling other types of glaucoma.

\section{Conclusion}

There was $36 \%$ mild, $26 \%$ moderate and $38 \%$ severe cases of POAG classified according to ICD 10. The mean value of contrast in mild, moderate and severe POAG was 1.4341, 1.3267 and 1.1326 respectively. There was decrease in contrast sensitivity with the severity of POAG. Thus the contrast sensitivity testing should be made mandatory in the evaluation of POAG patients which could help in early detection of glaucoma and also to monitor the progression of the disease as well as the efficacy of treatment.

\section{References}

1. Jesse Richman. Luciano L. Lorenzana, MD; Dara Lankaranian, MD; et al Importance of Visual Acuity and Contrast Sensitivity in Patients With Glaucoma. Arch Ophthalmol. 2010;128(12):1576-1582.

2. William J. Benjamin (Ed.). Borish's Clinical Refraction. $2^{\text {nd }}$ Edition. St. Lousi, Missouri. Butterworth Heinemann Elsevier ;2006 
3. Zulauf M, Flammer J. Correlation of spatial contrast sensitivity and visual fields in glaucoma. Graefes Arch Clin Exp Ophthalmol. 1993; 231:146-150.

4. Mutlukan E, Skarf B. Macular contrast sensitivity function correlates with automated threshold perimetry. In Wall M, Heijl A, eds. Perimetry Update 1996/1997, Proceedings of the XIIth International Perimetric Society Meeting. Kugler, Amsterdam 1997;243-250.

5. Tan JC, Spalton DJ, Arden GB. The effect of neodymium: YAG capsulotomy on contrast sensitivity and the evaluation of methods for its assessment. Ophthalmology. 1999;106:703-709.

6. Wood J, Lovie-Kitchin J. Evaluation of the Efficacy of Contrast Sensitivity Measures for the Detection of Early Primary Open-Angle Glaucoma. Optometry and Vision Science. 1992;69(3):175-181.

7. Wood JM, Lovie-Kitchin JE. Contrast sensitivity measurement in the detection of primary open angle glaucoma. In Mills R, Heijl A, eds. Perimetry Update 1990/1991, Proceedings of the IXth International Perimetric Society Meeting. Kugler, Amsterdam. 1991;497-502.

8. Mathai A, Thomas R, Braganza A, Maharajan S, George $\mathrm{T}$, Muliyil J. VISTECH contrast sensitivity testing in pri mary open angle glaucoma.

Indian J Ophthalmol. 1997;45:99-103.

9. Ross JE, Bron AJ, Clarke DD Contrast sensitivity and visual disability in chronic simple glaucoma. $\mathrm{Br} \mathrm{J}$ Ophthalmol. 1984;68(11):821-7.

10. Jacob T. Wilensky, MD, and (by invitation) Anjali Hawkins, MD, PhD comparison of contrast sensitivity, visual acuity, and Humphrey visual field testing in patients with glaucoma. Tr Am Ophth Soc. 2001;99:213218.
11. Hawkins A, Szlyk J, Ardickas Z, Alexander K, Wilensky J. Comparison of Contrast Sensitivity, Visual Acuity, and Humphrey Visual Field Testing in Patients with Glaucoma. Journal of Glaucoma. 2003;12(2):134-138.

12. McKendrick A, Sampson G, Walland M, Badcock D. Contrast Sensitivity Changes Due to Glaucoma and Normal Aging: Low-Spatial-Frequency Losses in Both Magnocellular and Parvocellular Pathways. Investigative Opthalmology \& Visual Science. 2007;48(5):2115.

13. Glovinsky Y, Quigley HA, Dunkelberger GR. Retinal ganglion cell loss is size dependent in experimental glaucoma. Invest Ophthalmol Vis Sci. 1991;32:484-491.

14. Quigley HA, Sanchez RM, Dunkelberger GR, L'Hernault NL, Baginski TA. Chronic glaucoma selectively damages large optic nerve fibers. Invest Ophthalmol Vis Sci. 1987;28:913-920.

15. Callaway EM. Structure and function of parallel pathways in the primate early visual system. J Physiol. 2005;566:13-19.

How to cite this article: Jha S. K, Ashwini K. V. Correlation of contrast sensitivity with the severity of primary open angle glaucoma. Indian J Clin Exp Ophthalmol. 2018;4(4):443-446. 